\title{
ON THE PROMPT SIGNALS OF GAMMA RAY BURSTS
}

\author{
P. Chen ${ }^{1}$, T. Tajima ${ }^{2}$ and Y. Takahashi ${ }^{3}$
}

\begin{abstract}
We introduce a new model of gamma ray burst (GRB) that explains its observed prompt signals, namely, its primary quasi-thermal spectrum and high energy tail. This mechanism can be applied to either assumption of GRB progenitor: coalescence of compact objects or hypernova explosion. The key ingredients of our model are: (1) The initial stage of a GRB is in the form of a relativistic quark-gluon plasma lava; (2) The expansion and cooling of this lava results in a QCD phase transition that induces a sudden gravitational stoppage of the condensed non-relativistic baryons and form a hadrosphere; (3) Acoustic shocks and Alfven waves (magnetoquakes) that erupt in episodes from the epicenter efficiently transport the thermal energy to the hadrospheric surface and induce a rapid detachment of leptons and photons from the hadrons; (4) The detached $e^{+} e^{-}$and $\gamma$ form an opaque, relativistically hot leptosphere, which expands and cools to $T \sim m c^{2}$, or $0.5 \mathrm{MeV}$, where $e^{+} e^{-} \rightarrow 2 \gamma$ and its reverse process becomes unbalanced, and the GRB photons are finally released; (5) The mode-conversion of Alfven waves into electromagnetic waves in the leptosphere provides a snowplow acceleration and deceleration that gives rise to both the high energy spectrum of GRB and the erosion of its thermal spectrum down to a quasi-thermal distribution. According to this model, the observed GRB photons should have a redshifted peak frequency at $E_{p} \sim \Gamma(1+\beta / 2) m c^{2} /(1+z)$, where $\Gamma \sim \mathcal{O}(1)$ is the Lorentz factor of the bulk flow of the lava, which may be determined from the existing GRB data.
\end{abstract}

\footnotetext{
Originally released as SLAC-PUB-8874 in 2001, this paper was never formally published.

${ }^{1}$ Department of Physics and Leung Center for Cosmology and Particle Astrophysics (LeCosPA), National Taiwan University, Taipei, Taiwan 10617 \& KIPAC, SLAC, Stanford University, CA 94035, USA; e-mail: pisinchen@phys.ntu.edu.tw

2 ZEST \& Ludwig-Maximilians-Universitat Munchen, Fakultat f. Physik, am Coulombwall 1, 85748 Garching, Germany

${ }^{3}$ Posthumous, Department of Physics, University of Alabama, Huntsville, AL 35899, USA
} 


\section{Introduction}

The fireball model of GRB proposed in $1980 \mathrm{~s}$ (Paczynski 1986; Goodman 1986; Shemi \& Piran 1990), which assumes a smooth expansion of the fireball, was later regarded as having difficulty to produce the high energy tail of the spectrum (Rees \& Meszaros 1992; Meszaros \& Rees 1993). This difficulty arises from the issue of baryon loading, where light particles (such as photons and electrons/positrons as well as neutrinos) cannot be easily detached from the opaque baryonic matter. It is generally believed that such a system would convert most of its energy into kinetic energy of the baryons rather than the luminosity. Indeed, Rees \& Meszaros 1992, 1993 focused on this feature as the major issue of GRB and proposed an alternative fireball shock model. In this model the exploding $e^{+} e^{-}$plasma has a bulk Lorentz factor $\Gamma \sim 10^{2}-10^{3}$ at a radius of $\sim 10^{5} \mathrm{~km}$. While this model addresses the issue of high energy tails, with a large Lorentz factor it remains a challenge to explain the spectral peak at several hundred $\mathrm{keV}$.

The typical spectrum of a GRB consists of a relatively broad, thermal-like spectrum, with the peak energy $E_{p}$ located at a few hundred $\mathrm{keV}$, which contributes more than half of its total luminosity. In the illustrative case of GRB 990510, activities of low energy spectrum $(<62 \mathrm{keV})$ precede the main sudden onset of the high energy spectrum $(>30 \mathrm{keV})$ by a few $10 \mathrm{~s}$ of seconds. In addition to the spectrum around the peak, a substantial fraction of the total luminosity is contributed from the high energy tail, which can be characterized by a power-law with a (negative) index $\sim 2-2.5$. In terms of the time structure, GRBs can be classified into two types: the short bursts that last for $\sim 1-10 \mathrm{sec}$ and the long bursts that last for tens to hundreds of seconds. It is interesting to note that while the time duration and profile vary widely over several orders of magnitude, the GRB spectra described above are remarkably universal. Much attention has been devoted to analyzing GRB afterglow as a result of an expanding fireball, which leads to important correspondence between the observational data and phenomenological models. However, a comprehensive understanding of the underlying mechanisms that produces such a fireball and the prompt signals are still lacking.

We suggest that the key to the understanding of GRB lies in its prompt signals, in particular the thermal portion of the spectrum. In this article we propose a new GRB model which provides a unified picture on the early-stage evolution and thus the mechanism that produces the prompt signals of GRBs. The key ingredients of our model are:

1. In the final stage of either compact-object coalescence or hypernova explosion, large fragments of hadron matter are ejected, most likely non-isotropic. Heated by the release of a large fraction of the systems gravitational potential energy, the hadrons are melted into quarks and gluons with temperature $\sim 200 \mathrm{MeV}$ and density $10^{38} \mathrm{~cm}^{3}$, like a molten lava. The bulk flow of such a lava, or hadrosphere, however, is only mildly relativistic.

2. The expansion and cooling of this lava results in a QCD (quantum chromodynamics) phase transition at a temperature $\sim 120 \mathrm{MeV}$ and density 
$\sim 2 \times 10^{37} \mathrm{~cm}^{3}$ that condensates the relativistic quarks and gluons into nonrelativistic baryons. These nonrelativistic baryons feel the strong gravity and stop their expansion. This results in the formation of a hardened hadrosphere boundary, analogous to the darkening of the lava surface.

3. Acoustic shocks and Alfven waves (magnetoquakes) that erupt in episodes from the epicenter efficiently transport the thermal energy to the hadrospheric surface and induce a rapid detachment of leptons and photons from the hadrons.

4. The detached $e^{+} e^{-}$and $\gamma$ form an opaque, relativistically hot leptosphere, which expands and cools to $T \sim m c^{2}$, or $0.5 \mathrm{MeV}$, below which $e^{+} e^{-} \rightarrow 2 \gamma$ and its reverse process become unbalanced, and the GRB thermal photons are released. The observed peak of this portion of the GRB spectrum is $E_{p} \sim \Gamma(1+\beta / 2) m c^{2} /(1+z)$, where $\Gamma \sim \mathcal{O}(1)$ is the Lorentz factor for the bulk flow of the lava, and $z$ is the GRB redshift factor.

5. The existence of a nonlinear $e^{+} e^{-}$plasma-mediated mode-conversion effect that converts Alfven waves into electromagnetic waves in the leptosphere. This process provides a novel snowplow acceleration and deceleration mechanism that produces both the high energy spectrum of GRB and the erosion of its thermal spectrum down to a quasi-thermal distribution.

Figure 1 is a schematic diagram that depicts our GRB model. In the following sections we elaborate these key points of our model in more details.

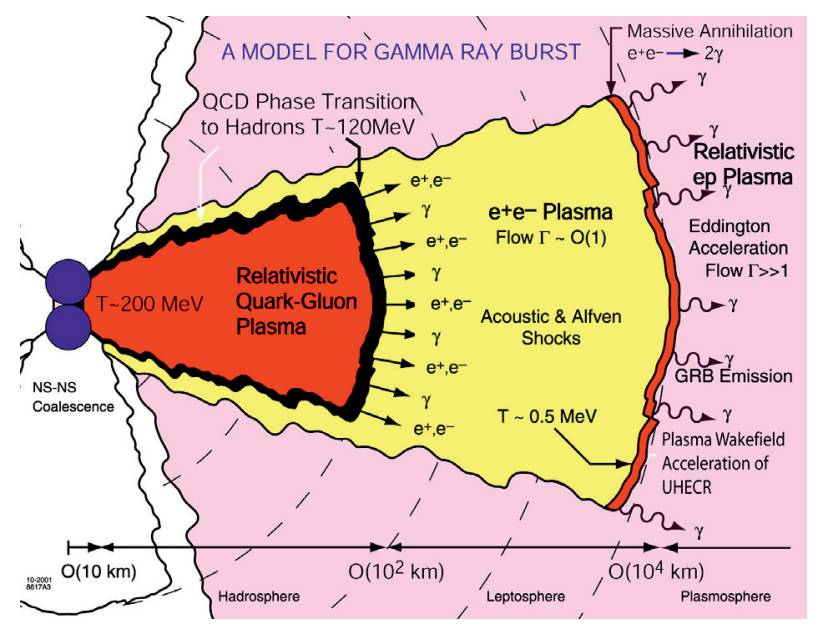

Fig. 1. A schematic diagram that depicts the various phases of the GRB dynamics in our model in the aftermath of the coalescence of a binary neutron star system. 


\section{Hadrosphere and QCD phase transition}

We assume that in the final stage of either compact-object coalescence or hypernova explosion, the tremendous concentration of energy triggers the eruption of large fragments of baryon matter. The density of baryon matter under such circumstance is comparable to that of a neutron star, i.e., $\sim 10^{38} \mathrm{~cm}^{3}$. Heated by the system's released gravitational energy, which can be as large as $\sim 0.1-0.3$ of the total rest mass of the system, such baryon fragments can gain a thermal energy, or temperature, $\sim 200 \mathrm{MeV}$. Under high temperature and density, one expects from quantum chromodynamics (QCD) that the baryon matter turns into a deconfined quark-gluon plasma (Alford 1998). A quantitative description of such QCD phase transition has been a major challenge to nuclear physicists. The standard approach is to invoke grand canonical ensemble (in which the particle number is not fixed), and therefore the relation between the temperature and the chemical potential. Nevertheless, we believe that the phase transition happens at temperature $T \geq 120 \mathrm{MeV}$ for zero baryon and at density $\rho \geq 10^{39} \mathrm{~cm}^{3}$ for much lower temperature (Liu 2001). Taking these conditions as our constraint and translating the chemical potential into an average particle density, we can parameterize the QCD phase boundary as

$$
\left(\frac{\rho}{\rho_{c}}\right)^{2}+\left(\frac{T}{T_{c}}\right)^{2}=1,
$$

where $\rho_{c} \sim 10^{39} \mathrm{~cm}^{3}$ and $T_{c} \sim 120 \mathrm{MeV}$. Clearly, the initial state of our system is in the quark-gluon phase.

Once quarks are deconfined at such energy-density, they are highly relativistic since their rest masses are as low as $m_{u} \sim 4 \mathrm{MeV}$ and $m_{d} \sim 7 \mathrm{MeV}$ for the up and down quarks, respectively. In this plasma there are about the same order of magnitude in the electron/positron (and neutrino) populations as well as thermal energies, since they are in (near) local thermal equilibrium with the relativistic quarks and gluons. Once this lava of quark-gluon plasma erupts, it adiabatically expands and cools. We call such a cluster the hadrosphere.

As the hadrosphere expands to the radius of $\sim 50 \mathrm{~km}$, the quark-gluon plasma density reduces to $\rho_{q-g} \sim 2 \times 10^{37} \mathrm{~cm}^{3}$. From thermodynamics the temperature and density are related by

$$
\rho^{1-\gamma} T=\text { const. }
$$

For relativistic particles, $\gamma=4 / 3$, and we find $T \propto \rho^{1 / 3}$. Since $\rho \propto 1 / V \propto 1 / R^{3}$, we have $T \propto 1 / R$. Thus the temperature drops to $T \sim 120 \mathrm{MeV}$ at this point. This is the temperature for QCD phase transition when the density is much lower than the critical one: $\rho \ll \rho_{c}$. Note, however, that in the case of NS-NS coalescence, the initial baryon density would be much higher than that of the nucleus, and therefore a much larger chemical potential. $T_{c}$, which is a function of both temperature and chemical potential, is thus much lower, at $\sim 10-20 \mathrm{MeV}$, and therefore the QCD phase transition is easier to reach. Once $T \sim T_{c}$, the quarks and gluons condensate into hadrons and turn nonrelativistic, which immediately feel the immense gravity 
and are thus gravitationally trapped. This gravitational capture of baryonic matter marks the boundary of the hadrosphere.

Note that such a quark-gluon explosion needs not be spherically symmetric, and may be irregular or even in chunks. Under the extreme high densities, the hadrosphere is highly opaque and poor in convection. Thus the quark-gluon plasma near the boundary first condensate into baryons while its interior is still molten. This is analogous to the darkening of the lava surface after erupted from the volcano, where the interior of the lava is still red-hot.

\section{Separation of photons and leptons from hadrosphere}

As mentioned in the Introduction, one seeming difficulty in the fireball model is the lack of a mechanism to efficiently transport the tremendous luminous energy near instantly across the baryonic matter. Given the extremely high density and therefore short mean-free-path in the fireball, the transport of energy through individual particle kinematics, i.e., thermal convection, would indeed be hard. This is the well-known problem of baryon loading. It may be overcome, however, by the transport of energy through collective plasma excitations.

In the final stage of compact-object coalescence or the collapse of supermassive star we expect the generation of strong acoustic waves (internal shocks) and Alfven waves (we may call this magnetoquakes). These waves are efficient mass and energy carriers (Holcomb \& Tajima 1991) in the interior of the hadrosphere as well as the leptosphere. For example, in the NS-NS or NS-BH coalescence, the violent perturbations of the strong magneticfield pressure of the host neutron stars $\left(B \sim 10^{12}-10^{13} \mathrm{G}\right)$ induces the excitation of magnetoquakes. As much as $\sim \mathcal{O}\left(10^{52}\right)$ erg of energy may be carried by these waves. Due to the compactness of the progenitor, the period of these magnetoquakes is about $\sim \mathcal{O}(100) \mu$ sec during each episode. As these shocks approach the boundary of the hadrosphere, the tortional as well as the compressional Alfven waves in the rapidly densitygraded stellar magnetosphere are expected to exhibit interesting and important properties (Takahashi et al. 2000). One is precisely the possibility of transport of energies from the epicenter to the hadrosphere boundary during each episode of magnetoquake. Another is the possibility of mode-conversion in the leptosphere. The density of the leptospheric $e^{+} e^{-}$plasma decreases rapidly due to its expansion. In such an environment the torsional Alfven waves can mode-convert themselves into the usual electromagnetic waves (Daniel \& Tajima 1998).

At the surface of hadrosphere, where the non-relativistic baryons are suddenly slowed down by self-gravitation as a result of QCD phase transition, the still highly relativistic leptons are freely radiating through the surface and their chemical potentials are negligible. Thus in close analogy with the standard blackbody emission process and according to the Stefan-Boltzmann law, $J=\alpha T^{4}$, where $\alpha=5.67 \times 10^{5} \mathrm{erg} / \mathrm{sec} / \mathrm{cm}^{2} / K^{4}$, the system can emit above $10^{52} \mathrm{ergs}$ in $10^{-8}$ second with a temperature of $120 \mathrm{MeV}$ and a radius of $50 \mathrm{~km}$. 


\section{Mode-conversion in leptosphere}

As mentioned earlier, the emitted $e^{+} e^{-}$and $\gamma$ are so dense that they are not freely propagating outward. With tremendous near-instant supply of electrons and positrons, the radiated $e^{+} e^{-}$pairs (as well as photons and neutrinos) will likely create shocks. As mentioned in the previous section, the internal acoustic and Alfvenic shocks can provide efficient energy transport as well as snowplow acceleration within the dense hadrosphere. In addition, the Alfven waves that continue to propagate across the leptosphere can induce a novel, linear and nonlinear phenomenon called mode-conversion. The density of the leptospheric $e^{+} e^{-}$plasma decreases rapidly due to its expansion. It has been observed in the particle-incell computer simulations that in such an environment the torsional Alfven waves can mode-convert themselves into ordinary electromagnetic waves (Kippen 1999). Furthermore, it was observed that inside such an opaque plasma a self-induced transparency occurs. Namely, a large number of energetic particles are plowed and accelerated in front of the Alfven wave, which are detached from the opaque, collisional bulk plasma.

When the mode-conversion occurs in the $e^{+} e^{-}$plasma, the converted EM waves proceed ahead of the Alfven waves and the snowplowed particles, forming an integrated overall trinity structure. This structure is capable of converting a large fraction of the wave energy (magnetoquake energy) into kinetic energies of the accelerated particles, as well as the heating of the bulk plasma. In our scenario this mechanism provides the basis of the production of the nonthermal high energy spectrum of GRB. The mechanism of this transport is analogous to snowplowing: particles are pushed forward in front of the shock waves. We note that such process can also decelerate those particles that are on the "wrong side" of the slope between episodes of magnetoquakes (Chen et al. 2002). Such stochastic processes will dilute the pure thermal spectrum into a quasi-thermal one.

\section{Quasi-thermal spectrum of GRB}

By the time when the leptosphere expands to a radius $\sim 10,000 \mathrm{~km}$ and cooled to below the two-photon pair production threshold, i.e., $T \sim m c^{2} \sim 0.5 \mathrm{MeV}$, the two-photon pair production and its reversed pair annihilation processes,

$$
e^{+} e^{-} \rightarrow 2 \gamma
$$

are out of balance, and the $e^{+} e^{-}$are largely annihilated into photons with a typical energy of $E_{p 0} \sim 0.5 \mathrm{MeV}$ in the rest frame of the bulk flow. The observed peak energy of the GRB thermal spectrum should therefore be

$$
E_{p} \sim \frac{\Gamma(1+\beta / 2)}{1+z} E_{p 0} \sim \frac{\Gamma(1+\beta / 2)}{1+z} m c^{2}
$$

where $z$ is the GRB redshift factor, $\beta^{2}=1-1 / \Gamma^{2}$ and $\Gamma$ is the Lorentz tactor of the bulk flow of the lava. As explained above, such initially thermal spectrum 
Table 1. Comparison of our model with observations based on the 7 GRB events from the BATSE catalog.

\begin{tabular}{||c||c|c|c|c||}
\hline BurstName & $E_{p}^{o b s}[\mathrm{keV}]$ & $z$ & $m c^{2} /(1+z)$ & Derived $\Gamma$ \\
\hline GRB 970508 & 481 & 0.84 & 278 & 1.3 \\
GRB 970825 & 230 & 0.96 & 261 & 0.9 \\
GRB 971214 & 156 & 3.41 & 116 & 1.1 \\
GRB 980703 & 370 & 0.97 & 259 & 1.2 \\
GRB 990123 & 550 & 1.60 & 197 & 1.9 \\
GRB 990506 & 450 & 1.20 & 232 & 1.4 \\
GRB 990510 & 174 & 1.62 & 195 & 0.9 \\
\hline
\end{tabular}

will be eroded to a quasi-thermal one due to the stochastic nature of snowplow acceleration-deceleration interplay under random phases of magneto quakes or shock waves.

To compare our model with observations, we take long burst GRBs with redshift factors identified from Piran et al. (2000), based on the BATSE data. There are 8 events where both the redshift and the spectral peak, $E_{p}$, have been identified. Among these 8 events on, GRB 980425, is discarded because it is very local $(z \sim 0.01)$ and its total luminosity fell sufficiently below the typical GRBs.

\section{Conclusion}

We have discussed the key features of our new model for GRB. Our scenario appears to be able to provide an explicit physical framework that can explain many of the GRB quasi-thermal spectrum characteristics. These include the release of $\sim 10^{52}$ erg of energy from a compact source, the promptness of such a release, and the origin of the GRB spectral peak as well as the high energy tail. Episodes of vibrations and eruptions of acoustic shocks and magnetoquakes, which should have a period of $\sim 100 \mu \mathrm{sec}$ during each burst, induce a fine structure within the overall duration of the prompt GRB signals. We have not discussed the physics in the outer plasmosphere (which is formed beyond the boundary of the leptosphere where positrons are essentially all annihilated). The existence of the plasmosphere, however, is in our view essential to another very important astrophysical phenomenon, namely the production of ultra-high energy cosmic rays (UHECR) beyond $10^{20} \mathrm{eV}$.

This work was supported by US Department of Energy, contract DE-AC03-76SF00515 (PC), DEFG03-96ER40954 (TT with UTA), W-7405-ENG-48 (TT with LLNL); and DE-FG-02-88ER41058 (YT); and by NASA, contract NAS898226 (YT). 


\section{References}

Alford, M., "New Possibilities for QCD at Finite Density", 1998, hep-lat/9809166 Chen, P., Tajima, T., \& Takahashi, Y., 2002, Phys. Rev. Lett., 89, 161101

Daniel, J., \& Tajima, T., 1998, ApJ, 498, 296

Goodman, J., 1986, ApJ, 308, L47

Holcomb, K.A., \& Tajima, T., 1991, ApJ, 378, 682

Kippen, R.M., 1999, GCN, 322

Liu, K.-F., private communications, April 2001

Meszaros, P., \& Rees, M.J., 1993, ApJ, 405, 278

Paczynski, B., 1986, ApJ, 308, L43

Piran, T., Jimenez, R., \& Band, D., 2000, The Energy Distribution of GRBs, in Gamma-Ray Bursts: 5th Huntsville Symposium, ed. R.M. Kippen et al., AIP Porc. 1-56396-947

Rees, M.J., \& Meszaros, P., 1992, MNRAS 258, 41

Shemi, A., \& Piran, T., 1990, ApJ, 365, L55

Takahashi, Y., Hillman, L.W., \& Tajima, T., 2000 in High-Field Science, ed. T. Tajima, K. Mima, \& H. Baldis (Kluwer Academic, NY), 171 